\title{
Quasicrystals and the Wulff-Shape
}

\author{
K. Böröczky, Jr., ${ }^{1}$ and U. Schnell ${ }^{2}$ \\ ${ }^{1}$ Mathematical Institute of the Hungarian Academy of Sciences, \\ Pf. 127, H-1364 Budapest, Hungary \\ carlos@cs.elte.hu \\ ${ }^{2}$ Mathematisches Institut, Universität Siegen, \\ D-57068 Siegen, Germany \\ schnell@mathematik.uni-siegen.de
}

\begin{abstract}
Infinite sphere packings give information about the structure but not about the shape of large dense sphere packings. For periodic sphere packings a new method was introduced in [W2], [W3], [Sc], and [BB], which gave a direct relation between dense periodic sphere packings and the Wulff-shape, which describes the shape of ideal crystals. In this paper we show for the classical Penrose tiling that dense finite quasiperiodic circle packings also lead to a Wulff-shape. This indicates that the shape of quasicrystals might be explained in terms of a finite packing density. Here we prove an isoperimetric inequality for unions of Penrose rhombs, which shows that the regular decagon is, in a sense, optimal among these sets. Motivated by the analysis of linear densities in the Penrose plane we introduce a surface energy for a class of polygons, which is analogous to the Gibbs-Curie surface energy for periodic crystals. This energy is minimized by the Wulff-shape, which is always a polygon and in certain cases it is the regular decagon, in accordance with the fivefold symmetry of quasicrystals.
\end{abstract}

\section{Introduction and Results}

Atomic dense packing is a fundamental property of crystals and quasicrystals and has been investigated for a long time (see [Se1]). In the last decade remarkable progress was made in the aperiodic case by various authors (see [Se2] and [VG]). These investigations consider infinite (space-filling) sphere packings without boundary effects. However, all packings in the real world are finite, and so there is the question whether the boundary and hence the shape of crystals and quasicrystals can also be described via dense sphere packings.

The theory of crystal growth is based on various energy principles. All these principles 
have in common that their minima of energy generate dense packings of spheres (atoms, ions). In particular the Gibbs-Curie surface energy plays an important role. For a crystal, whose $k$ faces $f_{1}, \ldots, f_{k}$ have given normal directions, the Gibbs-Curie surface energy is (see p. 124 of [L] or p. 304 of [Di]).

$$
\Phi=\sum_{i=1}^{k} \sigma_{i} f_{i},
$$

where $\sigma_{i}$ denotes the free surface energy per unit of the corresponding face. The crystallographer Wulff derived the equilibrium form, which minimizes $\Phi$ for all crystals with given volume and given facet normals. This form is given by the following construction (see [L] and [Di]). From a point inside the crystal, draw vectors in all normal directions, with length proportional to the corresponding $\sigma_{i}$. At the tip of each vector, construct an orthogonal plane. The equilibrium form, the so-called Wulff-shape, is the smallest convex region bounded by these planes. The construction shows that faces appear in the directions of greatest density. Later, more general minimization problems have been considered [T], [Wn].

The following packing density, introduced in [W1] and investigated in [BHW] and other papers, is flexible enough to model the Wulff-shape of crystals [W2], [W3], [Sc], [BB] and extremal crystals like whiskers and needles [W4]. We state the basic definitions and results. Let $B^{d}$ denote the unit ball in euclidean $d$-space $E^{d}(d \geq 2)$. Let $V$ denote the volume and $V\left(B^{d}\right)=\kappa_{d}$. For $X \subset E^{d}$ let $\operatorname{conv}(X)$ be its convex hull. Further, for a convex body (compact convex set with nonempty interior) $K$ and $\varrho \geq 0$ let $K+\varrho B^{d}=\left\{x+\varrho u: x \in K, u \in B^{d}\right\}$ be the parallel body of $K$ at the distance $\varrho$. In the following let $\mathcal{L}^{d}$ denote the set of lattices $L \subset E^{d}$ with $\operatorname{det} L \neq 0$ and for $L \in \mathcal{L}^{d}$ let $\mathcal{P}(L)$ be the set of polytopes $P$ with nonempty interior and facets orthogonal to suitable vectors of the polar lattice $L^{*}$ (see, e.g., [GL]).

If $C_{n}=\left\{c_{1}, \ldots, c_{n}\right\} \subset E^{d}$ is a discrete set, such that $C_{n}+B^{d}$ is a packing, then its parametric density is defined (see [W1] or [BHW]) by

$$
\delta_{\varrho}\left(B^{d}, C_{n}\right)=n \kappa_{d} / V\left(\operatorname{conv}\left(C_{n}+\varrho B^{d}\right)\right)
$$

where $\varrho>0$ is a parameter. For a convex body $K$ and a packing lattice $L$ for $K$ we write $\delta_{\varrho}\left(B^{d}, K\right)=\delta_{\varrho}\left(B^{d}, L, K\right)$ instead of $\delta_{\varrho}\left(B^{d}, K \cap L\right)$. In the case of lattice packings for any sequence $P_{n} \in \mathcal{P}(L)$, whose inradii tend to infinity, we have (see, e.g., p. 219 of [GL])

$$
\limsup _{n \rightarrow \infty} \delta_{\varrho}\left(B^{d}, P_{n}\right)=\kappa_{d} / \operatorname{det} L=: \delta_{L}
$$

where $\delta_{L}$ is the lattice packing density for $B^{d}$ and $L$. So no particular information is obtained and a refinement is needed. For this purpose the density deviation for $P \in \mathcal{P}(L)$ has been defined [W3] as

$$
\Delta_{L}(P, \varrho)=\lim _{\lambda \rightarrow \infty}(V(\lambda P))^{1 / d}\left\{\delta_{L}-\delta_{\varrho}\left(B^{d}, \lambda P\right)\right\}
$$

Since for small $\varrho$ a $(d-1)$-dimensional $P$ may be $\varrho$-denser than all polytopes in $\mathcal{P}(L)$, only

$$
\varrho>\varrho_{L}=\max \left\{(2\|u\|)^{-1}: u \in L^{*} \backslash\{0\}\right\}
$$


is considered. In [W3] it was proved that, for $P, Q \in \mathcal{P}(L)$ with $\Delta_{L}(P, \varrho)<\Delta_{L}(Q, \varrho)$, $P$ is, in a natural sense, asymptotically $\varrho$-denser than $Q$. Further, the density deviation can be determined (see formula (2.3) of [W3]) in terms of the $(d-1)$-dimensional facet volumes $f_{1}, \ldots, f_{k}$ of $P$ corresponding to the normals $u_{i} \in L^{*}$ :

$$
\Delta_{L}(P, \varrho)=\delta_{L}(V(P))^{(1-d) / d} \sum_{i=1}^{k} c_{i} f_{i}, \quad \text { with } \quad c_{i}=\varrho-\left(2\left\|u_{i}\right\|\right)^{-1} .
$$

It follows that the density deviation has the same form as the Gibbs-Curie surface energy in (1). Hence, minimizing the density deviation (i.e., maximizing the density) is equivalent to minimizing the free surface energy. Analogously to Wulff's construction, the minimum of $\Delta_{L}(P, \varrho)$ is attained for the Wulff-shape

$$
W(L, \varrho)=\left\{x \in E^{d}: u x \leq \varrho\|u\|-\frac{1}{2}, u \in L^{*} \text { primitive }\right\} .
$$

Only finitely many directions $u \in L^{*}$ contribute to $W(L, \varrho)$. So $W(L, \varrho)$ is a polytope, which depends only on $L$ and $\varrho$.

Since only few crystals are 1-atomic lattice packings (e.g., metals and rare gases), the density deviation has been generalized in $[\mathrm{Sc}]$ to arbitrary periodic structures with different sizes of spheres (atoms, ions). For the general periodic case analogous results have been shown and for certain crystal types (NaCl-type, ZnS-type, in particular the diamond) the Wulff-shape has been determined; according to reality.

The main goal of this paper is to apply the above concepts to nonperiodic sets in order to explain and also to model the growth and the shape of quasicrystals in terms of a finite packing density. Penrose tilings and their analogues have several properties of real quasicrystals. So they have been used as mathematical models explaining the structure of quasicrystals $[\mathrm{N}],[\mathrm{H}],[\mathrm{OK}]$. In this paper we restrict ourselves to the classical twodimensional Penrose tiling (see [P1], [P2], and [GS]). Of course, a more general approach is desirable. However, this would be beyond the scope of this paper. Nevertheless, the concepts introduced here can be applied to other nonperiodic tilings. In particular in a forthcoming paper [BS] the three-dimensional Penrose tiling will be considered. In certain cases we obtain as Wulff-shapes a dodecahedron and a rhombic triacontahedron; according to the shapes of single grains of certain real quasicrystals.

The Penrose tiling is obtained as a tiling of two rhombs, a large one with angles $2 \pi / 5$ and $3 \pi / 5$, and a small one with angles $\pi / 5$ and $4 \pi / 5$, with certain matching rules (see [GS] and [DB3]). Further, it can be produced by the process of inflation (see [DB3]). In one step of inflation the rhomb halves (see Fig. 1) are subdivided into smaller ones, which are rearranged according to certain rules (indicated by the arrows in Fig. 1) to obtain new rhombs. These are enlarged by the factor of the golden ratio $\tau=1 / 2(\sqrt{5}+1)=1.618 \ldots$. and have the original size again. Iteration of this step leads to a sequence of space-filling patches of the same shape. Another important method to produce quasiperiodic tilings is the projection method (see [DB1] and [OA]). A detailed description is given in Section 2. We use the inflation method as well as the projection method. In principle our concepts can be applied to any tiling, which is described by these methods.

In the following we let $\Pi$ be the Penrose plane and by $P$ we denote the family of vertices of the Penrose rhombs. For a subset $R \subset \Pi$ we denote by $A(R)$ and $P(R)$ the area and the perimeter of $R$. In Section 2 we consider the properties of the following family of subsets of $\Pi$. 

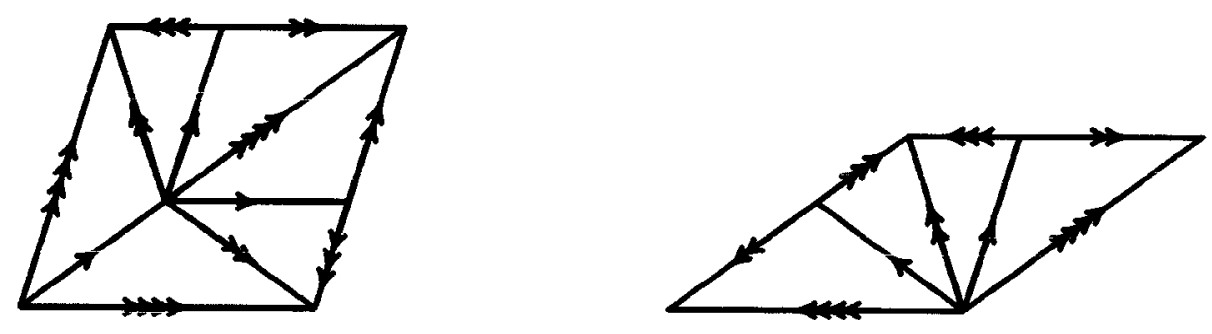

Fig. 1. Dissection of the Penrose rhombs.

Definition 1. A union $R$ of Penrose rhombs is called a $P$-set.

Furthermore, for a $P$-set $R$, we let $R_{n}$ be the resulting set after $n$ steps of inflation.

The two-dimensional infinite packing density of circles placed in the $P$-points has been determined (see, e.g., formula (1) of [OK]). By reasons of compatibility with the projection method we assume that the edge length of the rhombs is $\sqrt{\frac{2}{5}}$. It follows that the infinite (point-) density of $P$-points in $\Pi$ is $\delta=2(\tau+1) \sin \pi / 5 \sim 3.077$. In analogy to (4) we introduce the following limit.

Definition 2. For a $P$-set $R$ let

$$
\Delta(R, \varrho)=\lim _{n \rightarrow \infty}\left(A\left(\tau^{n} R\right)\right)^{1 / 2} \cdot\left(\delta-\#\left(P \cap \tau^{n} R\right) / A\left(\tau^{n} R+\varrho B^{2}\right)\right)
$$

be the density deviation for $R$ and the parameter $\varrho$.

Note that the integer multiplies are exchanged by the exponential factor $\tau^{n}$. In Section 2 we prove the following formula for the number \#( $\left.P \cap R_{n}\right)$ of $P$-points in $R_{n}$ for a $P$-set $R$. It is analogous to the Ehrhart formula for the number of lattice points (see p. 136 of [GL]). The change of the definition of $\Delta(R, \varrho)$ comes from the difference of these two formulas.

Theorem 1. Let $R$ be a P-set. Then

$$
\#\left(P \cap R_{n}\right)=\#\left(P \cap \tau^{n} R\right)=\delta A\left(\tau^{n} R\right)+\delta^{\prime} / 2 P\left(\tau^{n} R\right)+O(1),
$$

where $\delta^{\prime}=((2 \tau+1) / 5) \sqrt{\frac{5}{2}} \sim 1.339$.

$\delta^{\prime}$ is a certain linear density, which is considered in Section 3.

The Penrose tiling contains a regular decagon $T$ which is the union of five rhombs of each of both types (see Fig. 2).

In [RS] another nonperiodic tiling, the so-called pinwheel tiling, is considered and it is proved that there are unions of tiles arbitrarily close to a circle and so optimal with respect to the isoperimetric coefficient. It is mentioned there that for the Penrose tilings the regular decagon has the optimal isoperimetric coefficient. Since we did not find a reference, in Section 2 we give a proof for completeness. 


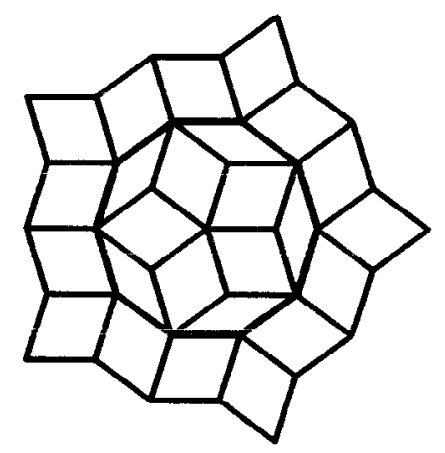

Fig. 2. The regular decagon is a $P$-set.

Theorem 2. Let $R$ be a $P$-set. Then

$$
A(R) \leq \sin (\pi / 5)((\tau+1) / 20) P(R)^{2},
$$

with equality if and only if $R$ is the regular decagon.

As a consequence of Theorem 1 and the Steiner formula for the parallel body (see p. 276 of [GL]) we can determine the density deviation for a $P$-set $R$ :

$$
\Delta(R, \varrho)=\delta\left(\varrho-\delta^{\prime} / 2 \delta\right) P(R)(A(R))^{-1 / 2} .
$$

From Theorem 2 it follows that the regular decagon has minimal density deviation.

Theorem 3. Let $R$ be a $P$-set and $\varrho>\delta^{\prime} /(2 \delta)$. Then

$$
\Delta(R, \varrho) \geq \Delta(T, \varrho),
$$

with equality if and only if $R$ is the regular decagon $T$.

We have seen that the densities of 2-planes play an important role for the surface energy of crystals. Hence we consider in our case linear (point-) densities of lines. These are defined as the limit of the ratio of the number of $P$-points in a line segment and its length. A look at the Penrose tiling shows that parallel lines do not necessarily have the same densities. We say that a direction $u \in \Pi$ is good if there is a line in direction $u$ with positive density of $P$-points. We prove in Section 3 that set of good directions is given by

$$
G=\left\{u=\alpha v^{4}+(\beta+\gamma \tau) v^{6}: \alpha, \beta, \gamma \in \mathbb{Z},(\alpha, \beta, \gamma)=1\right\},
$$

where $\left\{v^{4}, v^{6}\right\}$ is a certain basis of $\Pi$ and their angle is $2 \pi / 5$. Note that $G$ is countable and so has the same cardinality as lattices.

Generalizing the $P$-sets, we introduce the following notion.

Definition 3. A polygon $R \subset \Pi$ is called a $G$-polygon if its edges are in directions $u \in G$. 
Using the projection method, described in Section 2, we can determine for $u \in G$ the maximal density $\delta_{1}(u)$ of lines in direction $u$. Based on Diophantine approximation we can prove that there is a sequence of parallel lines in direction $u$, whose densities tend to $\delta_{1}(u)$ (see Section 3). Because of the distribution of the linear densities it is natural to introduce the Gibbs-Curie surface energy for $G$-polygons in analogy to (1) and (5).

Definition 4. Let $R$ be a $G$-polygon in $\Pi$ with edges in directions $u_{1}, \ldots, u_{k} \in G$ and lengths $f_{1}, \ldots, f_{k}$, then the Gibbs-Curie surface energy for the parameter $\varrho$ is defined as

$$
E(R, \varrho)=\sum_{i=1}^{k}\left(\varrho-\delta_{1}\left(u_{i}\right) /(2 \delta)\right) f_{i}
$$

The density deviation $\Delta(R, \varrho)$ can also be defined for $G$-polygons $R$ as in (7). Consequently we conjecture that a formula analogous to Theorem 1 also holds for $G$-polygons.

Conjecture. If $R$ is a $G$-polygon with sidelengths $f_{i}$ in directions $u_{i} \in G$, then

$$
\#\left(P \cap \tau^{n} R\right)=\delta A\left(\tau^{n} R\right)+\frac{1}{2} \tau^{n} \sum_{i=1}^{k} \delta_{1}\left(u_{i}\right) f_{i}+O(1) .
$$

Again, using the Steiner formula, it follows that:

Proposition. If the conjecture is true, then it holds for any G-polytope $R$ that

$$
\Delta(R, \varrho)=A(R)^{-1 / 2} \delta \sum_{i=1}^{k}\left(\varrho-\delta_{1}\left(u_{i}\right) /(2 \delta)\right) f_{i} .
$$

Hence, the density deviation, which is related to packing densities, and the surface energy are equivalent.

Now we want to minimize $E(R, \varrho)$ for fixed $\varrho$. The candidate for this minimum is the following Wulff-shape.

Definition 5. The Wulff-shape for the Penrose tiling for the parameter $\varrho>0$ is defined as

$$
W(\varrho)=\left\{z \in \Pi:\left|u^{\perp} z\right| \leq \varrho-\delta_{1}(u) /(2 \delta), \text { for all } u \text { in } G\right\},
$$

where $u^{\perp} \in \Pi$ with $\left\|u^{\perp}\right\|=1$ and $u^{\perp}$ orthogonal to $u$.

We only consider $\varrho$ with

$$
\varrho>\varrho^{*}=\sup _{u \in G}\left(\delta_{1}(u) /(2 \delta)\right)=\delta^{\prime} /(2 \delta)=0.217 \ldots
$$

This guarantees that $W(\varrho)$ is nonempty. 
Remark. For $\varrho<\varrho^{*}$ the asymptotic packing density of linear arrangements is better than the two-dimensional density $\delta$. So $\varrho^{*}$ can be considered as a critical radius or a sausage radius (see [W1] or [BHW]).

Since $W(\varrho)$ is the intersection of infinitely many half-planes it is not necessarily a polygon. However, with the help of Diophantine approximation we can prove that only finitely many $u \in G$ contribute to $W(\varrho)$.

Lemma 1. If $\varrho>\varrho^{*}$, then $W(\varrho)$ is a polygon.

An immediate consequence of Lemma 4 in [Sc] is that for $\varrho>\varrho^{*}$ the Wulff-shape minimizes the surface energy for $G$-polygons with fixed area.

Theorem 4. Let $\varrho>\varrho^{*}$ and let $R$ be a $G$-polygon with area $A(R)=A(W(\varrho))$. Then

$$
E(R, \varrho) \geq E(W(\varrho), \varrho),
$$

with equality only if $R$ is homothetic to $W(\varrho)$.

Finally we prove that for a certain range of $\varrho$ the Wulff-shape is the regular decagon, which already was the optimal $P$-set with respect to the density deviation (see Theorem 3).

Theorem 5. If $\varrho^{*}<\varrho<6 \varrho^{*}$, then $W(\varrho)$ is a regular decagon.

This result is in accordance with the fivefold symmetry of quasicrystals. So we really have a first hint that the growth and the shape of quasicrystals can be explained and modeled in terms of a finite packing density.

\section{Properties of $\boldsymbol{P}$-Sets}

First we describe how to obtain the Penrose tiling by projection. This will be used for the proof of Theorem 2 and to determine the linear densities in Section 3. The vertices of the Penrose rhombs are the projections of points of the integer lattice $\mathbb{Z}^{5}$ onto a plane $\Pi$, one of the two invariant planes $\Pi$ and $\Pi^{\prime}$ of the fivefold rotation about the diagonal $e$ of the unit cube in the Euclidean 5-space $C=\left\{x \in E^{5}: 0 \leq x_{1}, \ldots, x_{5} \leq 1\right\}$, which cyclically permutes the five coordinate axes. More exactly, consider the orthonormal basis

$$
\begin{aligned}
& u^{1}=1 / \sqrt{5} \cdot e=1 / \sqrt{5}(1,1,1,1,1)^{\mathrm{t}}, \\
& u^{2}=\sqrt{\frac{2}{5}}(1,(\tau-1) / 2,-\tau / 2,-\tau / 2,(\tau-1) / 2)^{\mathrm{t}}, \\
& u^{3}=\sqrt{\frac{2}{5}} \sin (\pi / 5)(0,-\tau,-1,1, \tau)^{\mathrm{t}}, \\
& u^{4}=\sqrt{\frac{2}{5}}(1,-\tau / 2,(\tau-1) / 2,(\tau-1) / 2,-\tau / 2)^{\mathrm{t}}, \\
& u^{5}=\sqrt{\frac{2}{5}} \sin (\pi / 5)(0,-1, \tau,-\tau, 1)^{\mathrm{t}}
\end{aligned}
$$




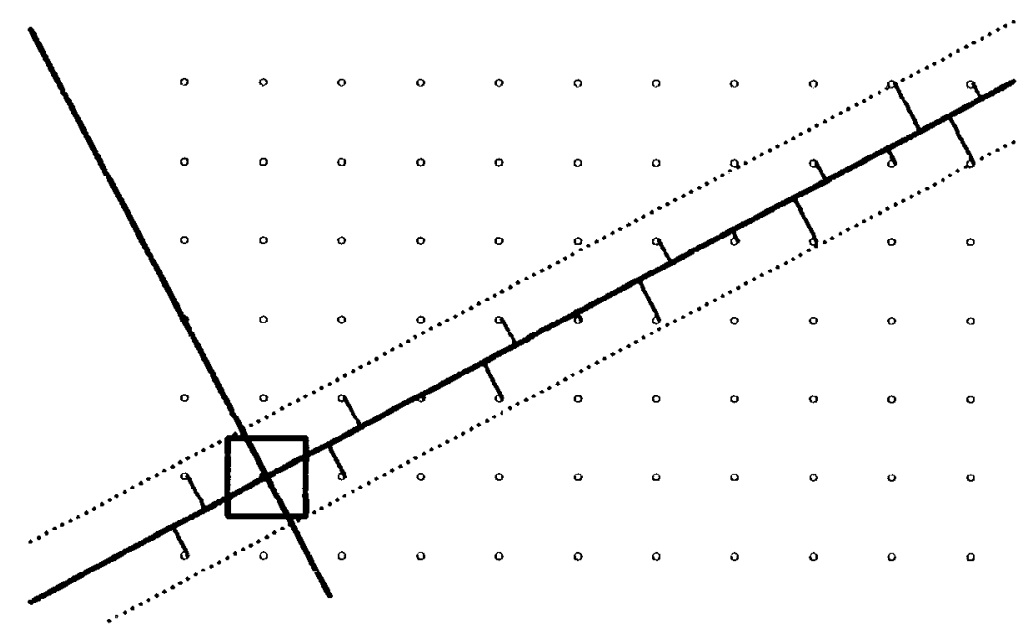

Fig. 3. A one-dimensional nonperiodic set obtained by the projection method.

of $E^{5}$ (the superscript $(\cdot)^{\mathrm{t}}$ indicates the transposed vector). We identify $\Pi=\operatorname{lin}\left(u^{4}, u^{5}\right)$ with the Penrose plane. Further, let $\Pi^{\prime}=\operatorname{lin}\left(u^{2}, u^{3}\right)$ and $E=\operatorname{lin}\left(u^{1}, u^{2}, u^{3}\right)$. For convenience we use in the following the orthogonal basis:

$$
\begin{gathered}
v^{1}=e, \quad v^{2}=(2, \tau-1,-\tau,-\tau, \tau-1)^{\mathrm{t}}, \quad v^{3}=(0,-\tau,-1,1, \tau)^{\mathrm{t}}, \\
v^{4}=(2,-\tau, \tau-1, \tau-1,-\tau)^{\mathrm{t}}, \quad v^{5}=(0,-1, \tau,-\tau, 1)^{\mathrm{t}} .
\end{gathered}
$$

Since the projection of $\mathbb{Z}^{5}$ is dense in $\Pi$, we only consider points whose projection into the orthogonal space $E$ lie in the interior of the orthogonal projection $C / E$, the so-called window $W$ ( $W$ is a rhombic icosahedron). The set $P$ of vertices of the Penrose rhombs is then

$$
P=\left((W+\Pi) \cap \mathbb{Z}^{5}\right) / \Pi \text {. }
$$

To illustrate the method we give a sketch of the projection of the two-dimensional integer lattice into a line with irrational slope (Fig. 3). Only lattice points which are contained in the strip determined by the square (whose projection into the orthogonal line is the window) are considered. The projections $e_{i} / \Pi$ of the unit vectors (and hence the edges of the rhombs) have length $\sqrt{\frac{2}{5}}$ and form a regular pentagon. The rhombs correspond to two different types of 2-faces of $C$ with area $A_{S}=\frac{2}{5} \sin (\pi / 5)$ and $A_{L}=\tau A_{S}$. For $k=1,2,3,4$ let

$$
W_{k}=(W \cap\{x: e x=k\}) / \Pi^{\prime} .
$$

$W_{1}$ is a regular pentagon, $W_{4}=-W_{1}$, and $W_{3}=-W_{2}=\tau W_{1}$.

Proof of Theorem 1. To obtain a formula for the number of $P$-points for a given $P$-set $R$ after $n$ steps of inflation, we follow the representation by De Bruijn [DB3] and take 


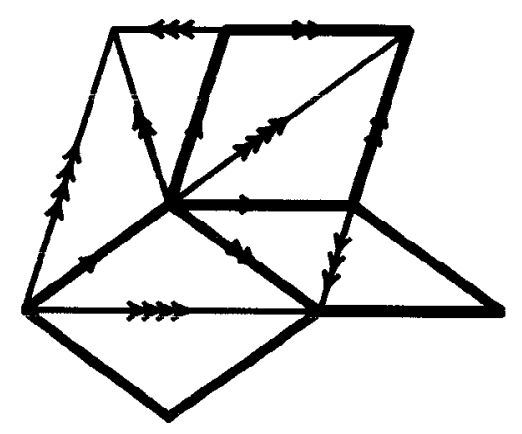

Fig. 4. An edge of type 1 generates edges of type 1 and 3 .

the different types of boundary edges into consideration. There are two different rhomb halves with six types of edges, the first three belonging to the large rhomb halves and the last three belonging to the small ones (see Fig. 1). Let type 1 and 4 be those where diagonals of the rhombs are boundary edges. Further let $x_{n}^{i}, i=1, \ldots, 6$, denote the number of boundary edges of type $i$ after $n$ steps of inflation. Figure 4 shows that a boundary edge of type 1 generates one edge of type 1 and one edge of type 3 in the next step. Analogous considerations for the five other types show that

$$
\begin{aligned}
& x_{n+1}^{1}=x_{n}^{1}+x_{n}^{2}+x_{n}^{5}, \\
& x_{n+1}^{2}=x_{n}^{3}, \\
& x_{n+1}^{3}=x_{n}^{1}, \\
& x_{n+1}^{4}=x_{n}^{3}, \\
& x_{n+1}^{5}=x_{n}^{4}, \\
& x_{n+1}^{6}=0 .
\end{aligned}
$$

If we define $q_{n}=x_{n}^{1}$, then we obtain $x_{n}^{3}=q_{n-1}, x_{n}^{2}=x_{n}^{4}=q_{n-2}, x_{n}^{5}=q_{n-3}$, and $q_{n}$ is given by the recursion

$$
q_{n+1}=q_{n}+q_{n-2}+q_{n-3} .
$$

Now let $a_{n}\left(b_{n}\right)$ be the number of large (small) half rhombs after $n$ steps. Then it follows from the inflation rules that

$$
a_{n+1}=2 a_{n}+b_{n}, \quad b_{n+1}=a_{n}+b_{n} .
$$

The area and the perimeter after $n$ steps are given by

$$
\begin{aligned}
A\left(R_{n}\right) & =a_{n} 1 / 2 A_{L}+b_{n} 1 / 2 A_{S}=A_{S} / 2\left(\tau a_{n}+b_{n}\right), \\
P\left(R_{n}\right) & =\sqrt{\frac{2}{5}}\left(x_{n}^{1} \tau+x_{n}^{2}+x_{n}^{3}+(\tau-1) x_{n}^{4}+x_{n}^{5}\right) \\
& =\sqrt{\frac{2}{5}}\left(\tau\left(q_{n}+q_{n-2}\right)+q_{n-1}+q_{n-3}\right) .
\end{aligned}
$$


Since $R_{n}=\tau^{n} R_{0}$ we further have

$$
\begin{aligned}
& A\left(R_{n}\right)=\tau^{2 n} A\left(R_{0}\right), \\
& P\left(R_{n}\right)=\tau^{n} P\left(R_{0}\right) .
\end{aligned}
$$

Let $c_{n}\left(d_{n}\right)$ be the number of large (small) rhombs, which are contained in $R_{n}$. Then

$$
\begin{aligned}
& c_{n}=1 / 2\left(a_{n}-x_{n}^{1}\right)=1 / 2\left(a_{n}-q_{n}\right), \\
& d_{n}=1 / 2\left(b_{n}-x_{n}^{4}\right)=1 / 2\left(a_{n}-q_{n-2}\right) .
\end{aligned}
$$

According to Euler's formula the number $\#\left(P \cap R_{n}\right)$ of $P$-points in $R_{n}$ is

$$
\#\left(P \cap R_{n}\right)=k_{n}+1-\left(c_{n}+d_{n}\right) .
$$

If $i_{n}$ and $r_{n}$ are the numbers of inner edges and boundary edges, respectively, then $k_{n}=i_{n}+r_{n}$ and $4\left(c_{n}+d_{n}\right)=2 i_{n}+r_{n}$ and hence

$$
\#\left(P \cap R_{n}\right)=r_{n}+2\left(c_{n}+d_{n}\right)-r_{n}+1-\left(c_{n}+d_{n}\right)=c_{n}+d_{n}+r_{n} / 2+1 .
$$

Together with $r_{n}=2 x_{n}^{1}+x_{n}^{2}+x_{n}^{3}+2 x_{n}^{4}+x_{n}^{5}=2 q_{n}+q_{n-1}+3 q_{n-2}+q_{n-3}$ it follows that

$$
\#\left(P \cap R_{n}\right)=1 / 2\left(a_{n}+b_{n}\right)+q_{n} / 2+q_{n-1} / 2 q_{n-2}+q_{n-3} / 2 k_{n}+1 .
$$

With a standard method for Lucas sequences (see, e.g., p. 39 of [PBH]) we conclude from (14) that there are constants $f_{1}, f_{2}$ such that

$$
\begin{aligned}
& a_{n}=\left\{1 / 5(2 \tau-1)\left(\tau a_{0}+b_{0}\right)\right\} \tau^{2 n}+f_{1}(2-\tau)^{n}, \\
& b_{n}=\left\{1 / 5(3-\tau)\left(\tau a_{0}+b_{0}\right)\right\} \tau^{2 n}+f_{2}(2-\tau)^{n},
\end{aligned}
$$

and so

$$
1 / 2\left(a_{n}+b_{n}\right)=2 \sin (\pi / 5)(\tau+1) A_{0} \tau^{2 n}+O(1) .
$$

For given $q_{-3}, q_{-2}, q_{-1}, q_{0}$ we obtain from (13) and the same method as used for $a_{n}$ and $b_{n}$ that there are constants $f_{3}, f_{4}$ such that

$$
\begin{aligned}
q_{n} & =\tau / 5\left(\tau\left(q_{0}+q_{-2}\right)+q_{-1}+q_{-3}\right) \tau^{n}+f_{3}(1-\tau)^{n}+\operatorname{Re}\left(f_{4} i^{n}\right) \\
& =P_{0} / 5 \sqrt{\frac{5}{2}} \tau^{n+1}+O(1) .
\end{aligned}
$$

Finally Theorem 1 follows from (15), (16), and (17).

Proof of Theorem 2. Let $x^{1}, x^{2} \in \mathbb{Z}^{5}$ such that $y^{i}=x^{i} / \Pi, i=1,2$, are $P$-points, and $x=x^{1}-x^{2}, y=y^{1}-y^{2}$. We want to give a bound for the ratio of $\left\|y^{1}-y^{2}\right\|$ and the length $\ell$ of the path from $y^{1}$ to $y^{2}$ along edges of the tiling. We have $\ell=\sqrt{\frac{2}{5}} \sum_{i=1}^{5}\left|x_{i}\right|=$ 
$\sqrt{\frac{2}{5}}\|x\|_{1}$. The point $x /\|x\|_{1}$ is contained in the unit ball with respect to $\|\cdot\|_{1}$, which is the crosspolytope $\mathrm{Cr}$. The projection $\mathrm{Cr} / \Pi$ is the regular decagon with circumradius $\sqrt{\frac{2}{5}}$. Hence

$$
\frac{\|y\|}{\ell} \leq \sqrt{\frac{5}{2}}\left\|\left(x /\|x\|_{1}\right) / \Pi\right\|=\frac{\sin (2 \pi / 5)}{\sin (2 \pi / 5+\alpha)}=\frac{\cos (\pi / 10)}{\cos (\pi / 10-\alpha)},
$$

where $\alpha=\alpha(y) \in[0, \pi / 10]$ is the smallest angle between $y$ and a projection of a unit vector $\pm e_{i}$. For any $y$ let $\beta(y)=\cos (\pi / 10) / \cos (\pi / 10-\alpha)$. Then it follows that

$$
\ell \geq \beta(y)\|y\| .
$$

For convex polygons $K$ with edges $y_{1}, \ldots, y_{r}$ we define

$$
\tilde{P}(K)=\sum_{i=1}^{r} \beta\left(y_{i}\right)\left\|y_{i}\right\| \text {. }
$$

To minimize $\tilde{P}$ we define

$$
W=\bigcap_{i=1}^{\infty}\left\{z: w_{i} z \leq \beta\left(y_{i}\right), \text { where }\left\|w_{i}\right\|=1 \text { and } w_{i} \perp y_{i}\right\} .
$$

Note that $y_{i}$ runs over all possible edges. The ten $y_{i}$ 's with $\beta\left(y_{i}\right)=1$ determine a regular decagon $T$. To prove that $W=T$, it suffices to show that $(\tan (\pi / 10), 1)^{\mathrm{t}} \in W$. This follows from $(\sin \alpha, \cos \alpha)(1, \tan \pi / 10)^{\mathrm{t}}=\beta(y)$. From Lemma 4 in [Sc] it follows that

$$
\frac{A(R)}{\tilde{P}(K)^{2}} \leq \frac{A(T)}{\tilde{P}(T)^{2}}=\frac{A(T)}{P(T)^{2}}=\sin \left(\frac{\pi}{5}\right) \cdot \frac{\tau+1}{20} .
$$

Now let $R$ be a $P$-set and let $K$ be its convex hull. Then $A(R) \leq A(K)$ and from (18) it follows that $P(R) \geq \tilde{P}(K)$. Together with (19) the assertion follows.

\section{The Linear Densities}

In this section we consider the density of $P$-points of lines in $\Pi$. Let $V$ be the $(5 \times 5)$ matrix with columns $v^{1}, \ldots, v^{5}$. Since $\mathbb{Q}[\tau]$ is a field it follows that the entries of $V^{-1}$ are contained in $\mathbb{Q}[\tau]$. For any $v \in \mathbb{Z}^{5}$ with $v=\sum_{i=1}^{5} \alpha_{i} v^{i}=V \cdot \alpha$ it follows from $\alpha=V^{-1} v$ that the coefficients are contained in $\mathbb{Q}[\tau]$. Hence any line in $\Pi$ which contains more than one $P$-point has a direction of the form

$$
\lambda v^{4}+\mu v^{5}, \quad \lambda, \mu \in \mathbb{Q}[\tau] .
$$

Because of the fivefold symmetry instead of $v^{5}$ we use the vector

$$
v^{6}=(\tau-1) / 2 v^{4}+\left(\tau-\frac{1}{2}\right) v^{5}=(\tau-1,-\tau, 2,-\tau, \tau-1)^{\mathrm{t}},
$$


which has an angle of $2 \pi / 5$ to $v^{4}$. So, it suffices to consider directions $u$ with

$$
u=\left(\alpha_{1}+\tau \alpha_{2}\right) v^{4}+\left(\beta_{1}+\tau \beta_{2}\right) v^{6}, \quad \alpha_{1}, \alpha_{2}, \beta_{1}, \beta_{2} \in \mathbb{Z} .
$$

Multiplication with $\alpha_{1}+\alpha_{2}-\tau \alpha_{2}$ shows that only directions from the set

$$
G=\left\{u=\alpha v^{4}+(\beta+\gamma \tau) v^{6}: \alpha, \beta, \gamma \in \mathbb{Z},(\alpha, \beta, \gamma)=1\right\}
$$

can have positive densities. If, for $u \in G, u=a+\tau b$, with $a, b \in \mathbb{Z}^{5}$, then

$$
\begin{aligned}
& a=(2 \alpha-\beta+\gamma,-\gamma,-\alpha+2 \beta,-\alpha-\gamma,-\beta+\gamma), \\
& b=(\beta,-\alpha-\beta-\gamma, \alpha+2 \gamma, \alpha-\beta-\gamma,-\alpha+\beta),
\end{aligned}
$$

and

$$
b-\tau a=(-\alpha \tau) v^{2}+(\gamma-\beta \tau) v^{7} \in \Pi^{\prime},
$$

where $v^{2}=(2, \tau-1,-\tau,-\tau, \tau-1)^{\mathrm{t}}$ and $v^{7}=(-\tau, \tau-1,2, \tau-1,-\tau)^{\mathrm{t}}$ are corresponding vectors in $\Pi^{\prime}$ with angle $2 \pi / 5$. It follows that the three-dimensional lattice

$$
L_{3}(u)=\mathbb{Z}^{5} \cap \operatorname{lin}\{a, b, e\}
$$

is projected onto the line $\mathbb{R} u$. Note that $L_{3}(u)=\operatorname{lin}\{u\}^{\perp} \cap \mathbb{Z}^{5}$, since $u$ is not a rational direction. Let $L_{2}(u)=\mathbb{Z}^{5} \cap \operatorname{lin}\{a, b\}$ and $\left\{a, b^{\prime}\right\}$ be a basis of $L_{2}(u)$ and $b^{\prime}=\eta a+v b$.

In the following we distinguish two cases:

(A) $\left\{a, b^{\prime}, e\right\}$ is a basis of $L_{3}(u)$,

(B) $\left\{a, b^{\prime}, e\right\}$ is not a basis of $L_{3}(u)$.

First we consider case (B). In this case there is a $v \in L_{3}(u)$ with $v e \not \equiv 0 \bmod 5$. Hence $(\mu v) e \equiv 1 \bmod 5$, for a suitable $\mu \in \mathbb{Z}$ and it follows the existence of a $c \in L_{3}(u)$ with $c e=1$. Then $\left\{a, b^{\prime}, c\right\}$ is a basis of $L_{3}(u)$ and $c=r a+s b+1 / 5 e$, for suitable $r, s$.

For $z \in \mathbb{Z}^{5}$ we consider the density of $P$-points in the line $g(z, u)=z / \Pi+\mathbb{R} u$. The set of lattice points which are projected into $g(z, u)$ is $z+L_{3}(u)$. For the density we consider those points $v=z+\lambda a+\mu b^{\prime}+\xi c, \lambda, \mu, \xi \in \mathbb{Z}$, with

(1) $\|v / \Pi-z / \Pi\| \leq \sigma$,

(2) $v / E \in W$.

For (2) necessarily ze $+\xi=k \in\{1,2,3,4\}$. For fixed $k \in\{1,2,3,4\}$ it follows from $a=1 / 5(3-\tau)(a+\tau b)+1 / 5(1-2 \tau)(b-\tau a)$ and $b=1 / 5(2 \tau-1)(a+\tau b)+1 / 5(3-$ $\tau)(b-\tau a)$ that (1) and (2) are equivalent to

$\left(1^{\prime}\right)\|1 / 5(a+\tau b)\{(3-\tau)(\lambda+\mu \eta+\xi r)+(2 \tau-1)(\mu \nu+\xi s)\}\| \leq \sigma$,

(2') $z / \Pi^{\prime}+1 / 5(b-\tau a)\{(1-2 \tau)(\lambda+\mu \eta+\xi r)+(3-\tau)(\mu \nu+\xi s)\} \in W_{k}$.

This is equivalent to

$\left(1^{\prime \prime}\right) \lambda(3-\tau)+\mu((3-\tau) \eta+(2 \tau-1) \nu) \in I_{\sigma}$,

$\left(2^{\prime \prime}\right) \lambda(1-2 \tau)+\mu((1-2 \tau) \eta+(3-\tau) \nu) \in I_{k}(z)$, 
where $I_{\sigma}$ and $I_{k}(z)$ are intervals of the length $(5 /\|a+\tau b\|) \cdot 2 \sigma$ and $(5 /\|b-\tau a\|) \cdot q_{k}(z)$ and $q_{k}(z)$ is the length of the intersection of the line $z / \Pi^{\prime}+\mathbb{R}(b-\tau a)$ with $W_{k}$.

Hence we count the integer points in a parallelogram $R_{\sigma, k}$ with increasing length and irrational slope. By a standard argument of uniform distribution (see [Se2]) the number of integer points in $R_{\sigma, k}$ is equal to $A\left(R_{\sigma, k}\right)+o(\sigma)$. So the ratio of the number of integer points in $R_{\sigma, k}$ and the length $2 \sigma$ is $((\tau+2) /(\|a+\tau b\| \cdot\|b-\tau a\| v)) q_{k}(z)$. With $q(z, u)=\sum_{i=1}^{4} q_{k}(z)$ it follows that the linear density in direction $u \in G$ is $\delta_{1}(z, u)=((\tau+2) /(\|a+\tau b\| \cdot\|b-\tau a\| v)) q(z, u)$. With $v=\operatorname{det} L_{2}(u) / \operatorname{det}(a, b)$ and $\|a+\tau b\| \cdot\|b-\tau a\|=\operatorname{det}(a+\tau b, b-\tau a)=(\tau+2) \operatorname{det}(a, b)$ it follows that

$$
\delta_{1}(z, u)=q(z, u) / \operatorname{det} L_{2}(u) .
$$

In case (A) the projection of $v=z+\lambda a+\mu b^{\prime}+\xi e$ can only be contained in a $W_{k_{0}}$ with $k_{0} \equiv z e \bmod 5$. The density can be determined in the same way as for case (B) and it follows that

$$
\delta_{1}(z, u)=q(z, u) / \operatorname{det} L_{2}(u) .
$$

Here $q(z, u)$ is 0 if $k_{0}=0$ and otherwise $q(z, u)$ is the length of the intersection of $z / \Pi^{\prime}+\mathbb{R}(b-\tau a)$ with $W_{k_{0}}$.

Remark. With the help of some number theoretical arguments one can prove that $\left\{a, b^{\prime}, e\right\}$ is a basis of $L_{3}(u)$ if and only if

$$
\alpha+\beta+3 \gamma \equiv 0 \bmod 5 \text { and } \alpha \neq \equiv \bmod 5 .
$$

For fixed $u=a+\tau b \in G$, with $a, b \in \mathbb{Z}^{5}$, consider

$$
q(u)=\sup _{z \in \mathbb{Z}^{5}} q(z, u) .
$$

In case (A) obviously $q(u)$ is not greater than the maximal chord $s(u)$ of $W_{2}$ in direction $b-\tau a$.

In case (B) it follows from $W_{3}=-W_{2}=\tau W_{1}=-\tau W_{4}$ that $q(u) \leq s(u)$, where $s(u)$ is $2(\tau+1)$ times the length of $W_{1} \cap(\mathbb{R}(b-\tau a))$.

Let $\left\{a^{1}, a^{2}, a^{3}\right\}$ be a basis of $L_{3}(u)$ and $a^{4}, a^{5} \in \mathbb{Z}^{5}$ such that $\left\{a^{1}, \ldots, a^{5}\right\}$ is a basis of $\mathbb{Z}^{5}$. Further, let

$$
\Lambda=\left\{\lambda\left(a^{4} / \Pi^{\prime}\right)+\mu\left(a^{5} / \Pi^{\prime}\right): \lambda, \mu \in \mathbb{Z}\right\} .
$$

From $\Lambda \cap \mathbb{R}(b-\tau a)=\emptyset$ it follows either that $\Lambda$ is a two-dimensional lattice in $\Pi^{\prime}$, where $(b-\tau a)$ is an irrational direction with respect to $\Lambda$, or $\Lambda$ is dense in a line $\neq \mathbb{R}(b-\tau a)$, i.e., the lengths of $a^{4} / \Pi^{\prime}$ and $a^{5} / \Pi^{\prime}$ are incommensurable. In both cases the optimal intersection $s(u)$ can be approximated by a suitable sequence $\left\{z_{n}\right\}$ in $\mathbb{Z}^{5}$ and it follows that $q(u)=s(u)$ (in fact, this can be done by diophantine approximation). Hence, for $u \in G$, there is a sequence of lines parallel to $\mathbb{R} u$ with linear densities tending to

$$
\delta_{1}(u)=q(u) / \operatorname{det} L_{2}(u) .
$$

Note that the edges during the inflation process in Section 2 determine such a sequence.

Motivated by this distribution of the linear densities, it is natural to define in analogy to periodic crystals (see (1) and (5)) the Gibbs-Curie surface energy as in (4). 
Next we consider some examples for the linear densities.

Examples. (1) There are ten directions $Z=\left\{ \pm e_{i} / \Pi\right\}$ with maximal $\delta_{1}$. These are of type (B). One member $u$ can be represented by $\alpha=1, \beta=\gamma=0$. Here $a=$ $(2,0,-1,-1,0)^{\mathrm{t}}, b=(0,-1,1,1,-1)^{\mathrm{t}}$, and $\{a, b\}$ is a basis of $L_{2}(u)$ with $\operatorname{det} L_{2}(u)=$ $2 \sqrt{5}$. With $q(u)=(4 \tau+3) \sqrt{\frac{2}{5}}$ it follows that $\delta_{1}(u)=\delta^{\prime}$ (see Theorem 1).

(2) Let $Z^{\prime}$ be the set of the ten directions in the middle of two members of $Z$. These are of type (A). One of them can be represented by $\alpha=\beta=\gamma=1$. Here $a=(2,-1,1,-2,0)^{\mathrm{t}}, b=(1,-3,3,-1,0)^{\mathrm{t}}$, and $\operatorname{det} L_{2}(u)=\operatorname{det}\left((1,0,0,-1,0)^{\mathrm{t}}\right.$, $\left.(0,1,-1,0,0)^{\mathrm{t}}\right)=2$. With $q(u)=\sqrt{\frac{2}{5}} \cdot 2 \tau \sin (2 \pi / 5)$ it follows that $\delta_{1}(u)=\delta^{\prime \prime}=$ $0.973 \ldots$.

\section{The Wulff-Shape}

Proof of Lemma 1. Since $W(\varrho)$ is compact it suffices to prove that $\varrho z \notin W(\varrho)$, for all $z \in \Pi$ with $\|z\|=1$. Because of the symmetry we only consider $z$ with $z=$ $\cos \xi u^{4}+\sin \xi u^{5}$ and $0<\xi<2 \pi / 5$. By diophantine approximation (see e.g., [GL]) there are infinitely many pairs $\left(\alpha_{n}, \beta_{n}\right) \in \mathbb{Z}^{2}$ with

$$
\left|\frac{\alpha_{n}}{\beta_{n}}+\frac{\tan \xi}{2 \sin (\pi / 5)}\right|<\frac{1}{\sqrt{5} \beta_{n}^{2}} .
$$

Let $u_{n}=\alpha_{n} v^{4}+\beta_{n} v^{5}=a_{n}+\tau b_{n} \in G$, with $a_{n}, b_{n} \in \mathbb{Z}^{5}$. Then $\operatorname{det} L_{2}\left(u_{n}\right) \leq$ $\left|\operatorname{det}\left(a_{n}, b_{n}\right)\right| \leq\left\|a_{n}\right\| \cdot\left\|b_{n}\right\|$ and so

$$
\delta_{1}\left(u_{n}\right) \geq \mu\left(\alpha_{n}+\beta_{n}\right)^{-2} \quad \text { for } \quad \mu>0 .
$$

Let $\eta_{n}$ be the angle between $u^{4}$ and $u_{n}^{\perp}$ and $\varphi_{n}=\eta_{n}-\xi$. Then $\tan \eta_{n}=-2 \sin (\pi / 5)$ $\alpha_{n} / \beta_{n}$. It follows with (26) that

$$
\begin{aligned}
(\varrho z) u_{n}^{\perp} & =\varrho \cos \varphi_{n} \geq \varrho-\varrho \sin ^{2}\left(\eta_{n}-\xi\right)=\varrho-\varrho\left(\tan \eta_{n}-\tan \xi\right)^{2} \cos ^{2} \eta_{n} \cos ^{2} \xi \\
& \geq \varrho-\left(\varrho \cos ^{2} \eta_{n} \cos ^{2} \xi\right) / \beta_{n}^{4} .
\end{aligned}
$$

With $\alpha_{n} / \beta_{n} \rightarrow \arctan \xi$ and $\eta_{n} \rightarrow \xi$ it follows that there is a constant $\gamma$ such that, for large $n$,

$$
(\varrho z) u_{n}^{\perp} \geq \varrho-\gamma /\left(\alpha_{n}+\beta_{n}\right)^{4}
$$

and with (27) it follows that there is a $u \in G$ with $(\varrho z) u^{\perp} \geq \varrho-\delta_{1}(u) /(2 \delta)$.

To prove Theorem 5 we first need the following upper bound for the linear densities.

Lemma 2. If $u \in G \backslash Z$, then

$$
\delta_{1}(u) \leq \delta^{\prime \prime}=0.973 \ldots
$$


Remark. The lemma states that the maximal linear density $\delta^{\prime}$ is attained for elements of $Z$ and the second best $\delta^{\prime \prime}$ is attained for elements of $Z^{\prime}$.

Proof. For $u \in Z$ or $u \in Z^{\prime}$ we have nothing to prove. Obviously it suffices to show that for $x, y \in \mathbb{Z}^{5}$ with $x / E, y / E \in W$ it follows that $\|x / \Pi-y / \Pi\| \geq(2 / 5(\tau+2))^{1 / 2}$ if $x / \Pi-y / \Pi$ is not in $Z \cup Z^{\prime}$. Since $\|x / E-y / E\|^{2} \leq 4$ it suffices to consider such $x, y$ with $\|x-y\|^{2} \leq 6$. For these finitely many vectors $x-y$ the assertion is easily verified.

Proof of Theorem 5. The ten directions in $Z$ determine a regular decagon. Because of the symmetry it suffices to prove that the vertex $x^{0}=\left(\varrho-\varrho^{*}\right)(\tan \pi / 10,1)^{\mathrm{t}}$ satisfies the conditions for all other $u \in G$. For any $u \in G$,

$$
u^{\perp} x^{0} \leq\left\|x^{0}\right\|=\left(\varrho-\varrho^{*}\right) / \cos (\pi / 10),
$$

and with Lemma 2 it suffices to prove that $\left(\varrho-\varrho^{*}\right) / \cos (\pi / 10) \leq \varrho-0.73 \varrho^{*}$. This is the case for $\varrho(1-0.73 \cos (\pi / 10)) /(1-\cos (\pi / 10)) \varrho^{*}=6.24 \ldots \varrho^{*}$.

\section{Acknowledgments}

We thank J. M. Wills and the referees for helpful comments.

\section{References}

[BB] U. Betke and K. Böröczky, Jr., Large lattice packings and crystals, Mathematika, to appear.

[BHW] U. Betke, M. Henk, and J.M. Wills, Finite and infinite packings, J. Reine Angew. Math. 453 (1994), 165-191.

[BS] K. Böröczky, Jr., and U. Schnell, Wulff shape for non-periodic arrangements, Lett. Math. Phys. 45 (1998), 81-94.

[DB1] A. De Bruijn, Algebraic theory of Penrose's non-periodic tilings in the plane, Nederl. Akad. Wetensch. Proc. Ser. A 84 (1981), 39-66.

[DB2] A. De Bruijn, Quasicrystals and their Fourier transforms, Nederl. Akad. Wetensch. Proc. Ser. A 89 (1986), 123-152.

[DB3] A. De Bruijn, Updown generation of Penrose patterns, Nederl. Akad. Wetensch. Indag. Math. 1(2), (1990), 201-219.

[Di] A. Dinghas, Über einen geometrischen Satz von Wulff über die Gleichgewichtsform von Kristallen, Z. Krist. 105 (1943), 304-314.

[GL] P.M. Gruber and C.G. Lekkerkerker, Geometry of Numbers, North-Holland, Amsterdam, 1987.

[GS] B. Grünbaum and G.C. Shephard, Tilings and Patterns, Freeman, San Francisco, CA, 1986.

[H] C. Henley, Sphere packings and local environments in Penrose tilings, Phys. Rev. 34 (1986), 797-816.

[L] M. von Laue, Der Wulffsche Satz für die Gleichgewichtsform von Kristallen, Z. Krist. 105 (1943), 124-133.

[N] D. R. Nelson, Quasicrystals, Scientific American 255 (1986), 32-41.

[OA] Z. Olami and S. Alexander, Quasiperiodic packing densities, Phys. Rev. 37 (1988), 3973-3978.

[OK] Z. Olami and M. Kleman, A two-dimensional aperiodic dense tiling, J. Phys. France 50 (1989), 19-33.

[P1] R. Penrose, The role of aesthetics in pure and applied mathematical research, Bull. Inst. Math. Appl. 10 (1974), 266-271.

[P2] R. Penrose, Pentaplexity, Eureka 39 (1978), 16-22. 
[PBH] A.N. Phllipou, G.E. Bergum, and A.F. Horodam (eds.), Fibonacci Numbers and Their Applications, Reidel, Dordrecht, 1986.

[RS] C. Radin and L. Sadun, The isoperimetric problem for Pinwheel Tilings, Comm. Math. Phys. 177 (1996), 255-263.

[Sc] U. Schnell, Periodic sphere packings and the Wulff-shape, Beiträge Algebra Geom., to appear.

[Se1] M. Senechal, Brief history of geometrical crystallography, in: Historical Atlas of Crystallography, J. Lima-de-Faria (ed.), Kluwer, Dordrecht, 1990.

[Se2] M. Senechal, Quasicrystals and Geometry, Cambridge University Press, Cambridge, 1995.

[T] J.E. Taylor, Crystalline variational problems, Bull. Amer. Math. Soc. 84 (1978), 568-588.

[VG] J.L. Verger-Gaugry, On a generalization of the Hermite constant, Period. Math. Hungar. 34 (1997), $153-164$.

[W1] J.M. Wills, Finite sphere packings and sphere coverings, Rend. Sem. Mat., Messina Ser. II 2 (1993), 91-97.

[W2] J.M. Wills, On large lattice packings of spheres, Geom. Dedicata 65 (1997), 117-126.

[W3] J.M. Wills, Lattice packings of spheres and Wulff-shape, Mathematika 86 (1996), 229-236.

[W4] J.M. Wills, Parametric density, online packings and crystal growth, Ren. Circ. Mat. Palermo 50 (1997), 413-424.

[Wn] S.J. Willson, A semigroup on the space of compact convex bodies, SIAM J. Math. Anal. 11 (1980), $448-457$.

Received June 1, 1997, and in revised form November 3, 1997. 